\title{
вмJ Global Health Digital health in South Africa: innovating to improve health
}

\author{
Yogan Pillay, Pakishe Aaron Motsoaledi
}

To cite: Pillay Y, Motsoaledi PA. Digital health in South Africa: innovating to improve health. BMJ Glob Health 2018;3:e000722. doi:10.1136/ bmjgh-2018-000722

Handling editor Seye Abimbola

Received 15 January 2018 Revised 23 January 2018 Accepted 24 January 2018

Check for updates

HIV, TB and MNCH, National Department of Health, South Africa, Pretoria, Gauteng, South Africa

Correspondence to

Dr Yogan Pillay;

ygpillay@gmail.com
In the postapartheid era, South Africa's public health system has been transformed from a dysfunctional system which perpetuated discrimination based by race and inequality into a deracialised, more comprehensive and integrated health system. ${ }^{1}$ In 2015 , rates of utilisation of health services across the continuum of care were the highest in sub-Saharan Africa: 94\% of pregnant women received antenatal care (ANC), $76 \%$ received the recommended four ANC visits, $96 \%$ delivered in a health facility, $97 \%$ had births attended by a skilled provider and $84 \%$ attended postnatal care within 2 days following birth. ${ }^{2}$ High rates of service utilisation have been underpinned by programmes like MomConnect which aim to empower pregnant and postpartum women with knowledge and bolster the utilisation of health services.

MomConnect was established by the National Department of Health in 2014 to register pregnancies and provide pregnant and postpartum women with twice-weekly health information text messages as well as access to a help desk for queries and feedback. ${ }^{34}$ Since its inception, MomConnect has grown to become one of the largest mHealth initiatives globally; by December 2017, it had cumulatively registered over 1.7 million pregnant women in over $95 \%$ (3300) of public health facilities nationally to receive short messaging service health information messages. It had also received more than 14000 spontaneously reported compliments to the help desk-eight times as many as the 1450 complaints received (personal communication Ms Jane Sebidi, Helpdesk Coordinator). The help desk has also impacted positively on improving the quality of care in the health system. ${ }^{5}$ Both complaints and compliments are sent to local coordinators to feedback to the health professional or facility to which they relate. This feedback is important to ensure that complaints are attended to and the complainant provided with report on action taken and that good work is acknowledged and encouraged.

In this journal supplement, we draw from experiences establishing, implementing and evaluating MomConnect in South Africa to provide critical learnings on the use of mobile and digital technology for global health in low-income and middle-income countries. We start with two editorials. The first, led by Dr Garrett Mehl and colleagues reflects on MomConnect from a global perspective, considering its implications for Universal Health Coverage in South Africa and globally. ${ }^{6}$ This perspective highlights two important features of MomConnect which offer a vehicle for pursuing universal health coverage: (1) its pregnancy registration feature and (2) the forethought in developing an interoperable digital health architecture with 'common good' elements for an interoperable digital national Health Information System. ${ }^{6}$ Both of these features set MomConnect apart from other digital health programmes, including maternal mobile messaging initiatives implemented elsewhere, and provide an important foundation for informing the future directionality of the programme and accommodating expansion into new areas. The second editorial, written by Dr Joanne Peter, provides a donor perspective on the history and evolution of MomConnect, considering the importance of public-private partnerships, including government stewardship and sustained donor funding. ${ }^{7}$

Following these editorials, we present a series of seven papers. The first two of these papers provide a background on the history of MomConnect and its architecture. In 'MomConnect: History, evolution, successes and challenges', Dr Peter Barron and colleagues provide an overview of MomConnect, detailing the groundwork, partnerships, stewardship and financing undertaken to establish and support it. ${ }^{4}$ Additional detail on the programme design, including registration and linkages to the health system, messaging, help desk and 
monitoring and evaluation features, help to describe key elements of the programme. In the next paper, Dr Chris Seebregts and colleagues provide an overview of the technical platform for MomConnect. ${ }^{8}$ Reliant on the use of open content, MomConnect's technical platform uses a health information exchange that can connect to any standards-compliant electronic front-end application as well as to any standards-compliant electronic back-end database. ${ }^{8}$ The end result is a platform which was designed and developed from the outset to be scalable and extendable across the country and serves as a reference implementation for South Africa's national eHealth architecture. $^{8}$

In the next four papers, we shift focus to analysis articles which draw on quantitative and qualitative methods. In the third paper, Dr Amnesty LeFevre and colleagues explore whether the messaging delivery component of MomConnect, including the underlying technical platform, performed as intended..$^{9}$ Through a series of descriptive analyses, they identify dropouts from point of contact with the health system during ANC clinics to successful registration and measure exposure to health information messages. ${ }^{9}$ While over $60 \%$ of all pregnant women attending antenatal clinics in the public sector were registered in 2017, there have been limitations in the current registration processes. There have been a large number of dropouts from registration and if rectified this would translate to an increase in coverage by 12\%-19\%—representing near universal coverage of all pregnant women attending ANC with access to a mobile phone. ${ }^{9}$ Findings from analyses on message delivery suggest that an estimated $80 \%$ of intended messages were successfully sent—a figure well above that reported elsewhere for maternal messaging programmes. ${ }^{10}$ In the fourth paper, Dr Alexa Heekes and colleagues demonstrate the feasibility and value of linking MomConnect data with records collected through a province-wide health information exchange in the Western Cape Province of South Africa. ${ }^{11}$ Pregnant women registered with MomConnect had fewer adverse pregnancy outcome events such as stillbirths. However, data suggest that those at lower risk of adverse pregnancy outcomes were more likely to register for MomConnect. ${ }^{11}$

The fifth and sixth papers in the series focus on MomConnect's helpdesk. In the fifth paper, Ms Khou Xiong and colleagues explored trends in the utilisation of the helpdesk, determining that $8 \%$ of MomConnect's registered users utilised the helpdesk. ${ }^{12}$ Helpdesk use was primarily to seek maternal health information and where feedback about health services was provided, there were significantly more compliments than complaints. ${ }^{12}$ In the second help desk paper, Dr Matt Engelhard and colleagues evaluate the need for and feasibility of automated message triage to improve help desk responsiveness to high-priority messages. ${ }^{13}$ Using keyword matching and a Naive Bayes classifier, low prevalence high-priority messages related to the disrespect and abuse of women during childbirth were effectively identified, which could support automated triage to improve handling of high-priority messages moving forward. ${ }^{13}$ The seventh and final paper in this series led by Dr Donald Skinner and colleagues explores user perceptions of and the use of MomConnect health information messages through qualitative in-depth interviews and focus group discussions. ${ }^{14}$ Findings suggest that MomConnect users were enthusiastic about the messages, reporting that the information was useful and empowering, with some saving the messages to use as a resource or to share with others. ${ }^{14}$

Finally, we conclude our series with a final editorial on 'Taking digital health innovation to scale in South Africa: ten lessons from MomConnect'. ${ }^{15}$ In this last paper, Dr Joanne Peter and colleagues highlight lessons across domains of leadership and partnerships, technology and architecture, content and user engagement, financial health and monitoring and evaluation. ${ }^{15}{ }^{16}$ In view of the limited number of digital health programmes which have been scaled globally and which are government led, the emerging lessons are anticipated to provide vital insights for other countries similarly considering the pros and cons of technology use in the health sector.

This is the first journal supplement of its kind on a national level digital health programme. MomConnect is one of the only five mobile health information messaging programmes to have scaled to over 1 million beneficiaries and the only programme to have attained population level coverage of $>60 \% .{ }^{15}$ While a multitude of factors underpin the programme's successful implementation, perhaps none are more important than the pregnant and postpartum women in South Africa who form the backbone of our communities, and the health workers who work tirelessly to register women and provide vital health services. The commitment to technology use in the health sector is a critical component of the Department of Health's vision to embrace innovative strategies for improving health services. By reflecting on components of MomConnect, we hope to catalyse discussion on how to optimally design, prioritise, plan and successfully implement a new wave of digital health solutions in South Africa and in other low-resource settings where the disease burden is acute.

Acknowledgements The support provided by John Snow, Inc. (JSI) in the President's Emergency Plan for AIDS Relief (PEPFAR) and United States Agency for International Development (USAID)-funded MEASURE Evaluation Strategic Information for South Africa (MEval-SIFSA) project to enable this publication is acknowledged with gratitude.

Contributors This editorial was written by YP and PM.

Funding This study was supported by the project MEASURE Evaluation-Strategic Information for South Africa, a President's Emergency Plan for AIDS Relief (PEPFAR) initiative.

Competing interests None declared.

Provenance and peer review Not commissioned; internally peer reviewed.

Data sharing statement No additional data are available. 
Open Access This is an Open Access article distributed in accordance with the terms of the Creative Commons Attribution (CC BY 4.0) license, which permits others to distribute, remix, adapt and build upon this work, for commercial use, provided the original work is properly cited. See: http://creativecommons.org/ licenses/by/4.0/

(C) Article author(s) (or their employer(s) unless otherwise stated in the text of the article) 2018. All rights reserved. No commercial use is permitted unless otherwise expressly granted.

\section{REFERENCES}

1. Coovadia $H$, Jewkes $R$, Barron $P$, et al. The health and health system of South Africa: historical roots of current public health challenges. Lancet 2009;374:817-34.

2. National Department of Health $(\mathrm{NDoH})$, Statistics South Africa (Stats SA) South African Medical Research (SAMRC) and ICF. South Africa demographic and health survey 2016. Pretoria, South Africa, and Rockville, Maryland, USA: National Department of Health (NDoH), Statistics South Africa (Stats SA) South African Medical Research (SAMRC) and ICF, 2017.

3. Peter JE, Barron P, Pillay Y. Using mobile technology to improve maternal, child and youth health and treatment of HIV patients. $S$ Afr Med J 2015;106:3-4.

4. Barron P, Peter J, LeFevre A, et al. Mobile health messaging service and helpdesk for South African mothers (MomConnect): history, successes and challenges. BMJ Global Health 2018;3(Suppl 2):e000559.

5. Barron P, Pillay Y, Fernandes A, et al. The MomConnect mHealth initiative in South Africa: early impact on the supply side of $\mathrm{MCH}$ services. J Public Health Policy 2016;37(Suppl 2):201-12.

6. Mehl G, Tamrat T, Bhardwaj S, et al. The digital health vision: could MomConnect provide a pragmatic starting point for achieving universal health coverage in South Africa and elsewhere. BMJ Global Health 2018;3(Suppl 2):e000626.
7. Peter J. Achieving scale, sustainability and impact: a donor perspective on a mobile health messaging service and helpdesk for South African mothers (MomConnect). BMJ Global Health 2018;3(Suppl 2):e000562.

8. Seebregts C, Dane P, Parsons A, et al. Designing for scale: optimising the health information system architecture of MomConnect in South Africa. BMJ Global Health 2018;3(Suppl 2):e000563.

9. LeFevre A, Dane P, Copley C, et al. Unpacking the exposure to mobile health information messages for mothers in South Africa: evidence on messaging reach and platform effectiveness. BMJ Global Health 2018;3(Suppl 2):e000583.

10. LeFevre AE, Mohan D, Hutchful D, et al. Mobile technology for community health in Ghana: what happens when technical functionality threatens the effectiveness of digital health programs? BMC Med Inform Decis Mak 2017;17:27.

11. Heekes A, Tiffin N, Dane P, et al. Self-enrolment antenatal health promotion data as an adjunct to maternal clinical information systems in the Western Cape Province of South Africa. BMJ Global Health 2018;3(Suppl 2):e000565.

12. Xiong K, Kamunyori J, Sebidi J, et al. The MomConnect Help Desk: a secondary data analysis to determine how the help desk messages are contributing to improved maternal health service delivery in South Africa. BMJ Global Health 2018;3(Suppl 2):e000578.

13. Engelhard M, Copley C, Watson J, et al. Optimizing the MomConnect Helpdesk in South Africa: towards automated message triage. BMJ Global Health 2018;3(Suppl 2):e000567.

14. Skinner D, Delobelle P, Pappin M, et al. User assessments and the use of information from MomConnect by mothers in South Africa. BMJ Global Health 2018;3(Suppl 2):e000561.

15. Peter J, Benjamin P, LeFevre A, et al. Taking digital health innovation to scale in South Africa: ten lessons from MomConnect. BMJ Global Health 2018;3(Suppl 2):e000592.

16. WHO, JHU-GMI, UNF. The MAPS toolkit: mHealth assessment and planning for scale. Geneva: WHO Department of Reproductive Health and Research, 2015. 